\section{Benign metastasizing leiomyoma}

W. V. Houck, ${ }^{1}$ T. J. Broderick, ${ }^{2}$ S. A. Cohen,${ }^{3}$ N. M. Cohen ${ }^{1}$

${ }^{1}$ Section of General Thoracic Surgery; Division of Cardiothoracic

Surgery, Department of Surgery, Box 980068, Medical College of

Virginia Hospitals and Physicians of Virginia Commonwealth Uni-

versity Health System, Richmond, VA 23298-0068, USA

${ }^{2}$ Division of General Surgery, Department of Surgery, Medical College of Virginia Hospitals and Physicians of Virginia Commonwealth University Health System, Richmond, VA 23298, USA

${ }^{3}$ Department of Obstetrics and Gynecology, Medical College of Virginia Hospitals and Physicians of Virginia Commonwealth University Health System, Richmond, VA 23298 , USA

Received: 9 August 2001/Accepted in final form: 6 November 2001/ Online publication: 8 February 2002

DOI: $10.1007 / \mathrm{s} 00464-001-4138-5$

Abstract

Benign metastasizing leiomyoma is a rare clinical entity that has been described in several previous reports. Although the exact pathophysiology of the disease is unknown, two predominant theories exist: (1) metastasis from an existing leiomyoma (commonly seen with uterine leiomyoma) or (2) multicentric leiomyomatous growths rather than actual metastases. We present an interesting case in which several elements of the patient's history complicated the differential diagnosis.

Key words: Leiomyoma - Pulmonary nodules Oncology - Multiple endocrine neoplasms

Correspondence to: N. M. Cohen

\section{Necrotizing fasciitis with Clostridium perfringens after laparoscopic cholecystectomy}

\section{Case report and review of the literature}

R. P. Mittermair, ${ }^{1}$ W. Schobersberger, ${ }^{2}$ F. Allerberger, ${ }^{3}$ R. Peer, ${ }^{4}$ W. Hasibeder ${ }^{2}$

${ }^{1}$ Department of General Surgery, University Hospital Innsbruck.

University of Innsbruck, Anichstrasse 35, A-6020 Innsbruck, Austria

${ }^{2}$ Division for General and Surgical Intensive Care Medicine,

University Hospital Innsbruck, University of Innsbruck, Austria

${ }^{3}$ Institute for Hygiene, University of Innsbruck, Austria

${ }^{4}$ Department of Radiology, University Hospital Innsbruck, University of Innsbruck, Austria

Received: 3 September 2001/Accepted in final form: 6 September 2001/ Online publication: 14 January 2002

DOI: $10.1007 / \mathrm{s} 00464-001-4232-8$

Abstract

Necrotizing fasciitis is a rapidly progressive infection of the fascia and subcutaneous tissues accompanied by a high mortality rate approaching $80 \%$ to $100 \%$. Factors that predispose patients to this life-threatening complication include obesity, malnutrition, malignancy, chronic alcoholism, drug abuse, peripheral vascular disease, diabetes mellitus, and immunosuppressive therapy. The pathomechanisms for the development of this rare disease still remain unclear. We report a case of necrotizing fasciitis with Clostridium perfringens after laparoscopic cholecystectomy. The patient left the hospital 5 months after admission. Early recognition based on clinical signs (pain, asymmetric abdominal thickening, crepitus) and computed tomography scanning (gas dissection along fascial planes), in conjunction with prompt, aggressive surgical therapy and debridement of all devi- talized tissue, high-dose antibiotic therapy, and therapy at the intensive care unit, appears to afford patients the best chance of survival.

Key words: Necrotizing fasciitis - Clostridium perfringens - Laparoscopic cholecystectomy

Correspondence to: R. P. Mittermair

\section{Byler disease associated with acute cholecystitis}

\section{Case report and review of the literature}

M. Abbas, Y. Hussain, K. Al-Beloushi

Department of Surgery, Farwania Hospital, State of Kuwait

Received: 19 July 2001/Accepted in final form: 15 August 2001/Online publication: 14 January 2002

DOI: $10.1007 / \mathrm{s} 00464-001-4224-8$

Abstract

A case of Byler disease, which has a rare incidence of 1 in 90000 , is reported. A 21 -year-old woman known to have the disease since childhood presented with acute cholecystitis and underwent laparoscopic cholecystectomy. To the best of our knowledge, after a review of the English literature, this is the second patient with Byler disease to live beyond the second decade of life since the disorder was first described by Clayton 30 years ago, and the first case associated with acute cholecystitis that was managed successfully by laparoscopic cholecystectomy.

Key words: Byler disease - Acute cholecystitis Laparoscopic cholecystectomy Correspondence to: M. Abbas

\section{Endoscopic extraperitoneal repair of a Grynfeltt hernia}

R. R. Postema, ${ }^{1,2}$ H. J. Bonjer ${ }^{1}$

'Department of Surgery, University Hospital Rotterdam, Dr. Molewaterplein 60,3015 GJ Rotterdam, The Netherlands

${ }^{2}$ Department of Pediatric surgery, University Hospital Rotterdam, Dr. Molewaterplein 60,3015 GJ Rotterdam, The Netherlands

Received: 28 June 2001/Accepted in final form: 15 August 2001/Online publication: 14 January 2002

DOI: $10.1007 / \mathrm{s} 00464-001-4218-6$

Abstract

There are three types of lumbar hernia: congenital, acquired, and incisional hernias. Acquired hernia can appear in two forms: the inferior (Petit) type and the superior type, first described by Grynfeltt in 1866 . We report endoscopic extraperitoneal repair of a Grynfeltt hernia. A 46-year-old woman presented with a painful swelling in the left lumbar region that had caused her increasing discomfort. The diagnosis of Grynfeltt's hernia was made, and she underwent surgery. With the patient in a left-side decubitus position, access to the extraperitoneal space was gained by inserting a $10-\mathrm{mm}$ inflatable balloon trocar just anteriorly to the midaxillary line between the 12 th rib and the superior iliac crest through a muscle-splitting incision into the extraperitoneal space. After the balloon trocar had been removed a blunt-tip trocar was inserted. Using two 5-mm trocars, one above and another below the $10-\mathrm{mm}$ port in the midaxillary line, the hernia could be reduced. A poly- 